\title{
Mammography correlates to better survival rates in breast cancer patients: a 20-year experience in a University health institution
}

\author{
Cristóbal Maiz ${ }^{1}$, Fernando Silva², Francisco Domínguez ${ }^{1}$, Héctor Galindo², Mauricio Camus ${ }^{1}$, Augusto León ${ }^{1}$, David Oddó ${ }^{3}$, \\ Alejandra Villarroel ${ }^{3}$, Dravna Razmilic ${ }^{4}$, María Elena Navarro ${ }^{4}$, Lidia Medina ${ }^{5}$, Tomás Merino², Eugenio Vines ${ }^{2}$, José Peña ${ }^{2}$, \\ Daniela Maldonado ${ }^{1}$, Mauricio P. Pinto ${ }^{2}$, Francisco Acevedo² and César Sánchez ${ }^{2}$
}

\begin{abstract}
${ }^{1}$ Departament of Oncological and Maxillofacial Surgery, School of Medicine, Pontificia Universidad Católica de Chile, 8330032 Santiago, Chile ${ }^{2}$ Departament of Hematology and Oncology, School of Medicine, Pontificia Universidad Católica de Chile, 8330032 Santiago, Chile ${ }^{3}$ Departament of Anatomic Pathology, School of Medicine, Pontificia Universidad Católica de Chile, 8330032 Santiago, Chile ${ }^{4}$ Departament of Radiology, School of Medicine, Pontificia Universidad Católica de Chile, 8330032 Santiago, Chile ${ }^{5}$ Cancer Center 'Nuestra Señora de la Esperanza', Pontificia Universidad Católica de Chile, 8330032 Santiago, Chile
\end{abstract}

\section{Abstract}

Breast cancer $(\mathrm{BC})$ is the most common malignancy in women. We retrieved medical records from $>2,000$ Chilean BC patients over the 1997-2018 period. The objective was to assess changes in clinical presentation or prognosis of our patients throughout these 20 years of practice. Although most variables did not display significant variations, we observed a progressive increase in stage IV BC over this period. Our data showed that tumour stage III/IV or HER2-enriched subtype tumours were associated with poorer prognosis. In contrast, we found that patients diagnosed by mammography had better overall survival. We speculate that better screenings and more sensitive imaging could explain the unexpected rise in stage IV cases. Our results support mammography screenings as an effective measure to reduce BC-related mortality.

Keywords: breast neoplasia, prognosis, survival, mammography, advanced breast cancer

\section{Introduction}

Worldwide, breast cancer (BC) is a high incidence cancer among women. In Chile, it is women's leading cause of cancer death [1]. Reports indicate an increase in BC incidence $[1,2]$ in recent decades. Several factors could explain this observation: changes in lifestyle and better screenings along with an aging population.

The widespread use of mammography, better treatments and supportive care have allowed a progressive improvement in prognosis and a sustained $1 \%-2 \%$ increase in survival every year over the last 30 years in developed countries [1, 3].

Evidently, changes in diagnostic methods and staging of the disease [4] may be an interpretation bias in cancers associated to good prognosis (such as breast). This study analysed the clinical features of BC patients diagnosed at our institution over 20 years. Our data indicates a progressive increase in stage IV cases over time. Importantly, we confirmed that the diagnosis by mammography was associated with reduced mortality.
Correspondence to: Cesar Sanchez Email: csanchez@med.puc.cl

ecancer 2020, 14:1005

https://doi.org/10.3332/ecancer.2020.1005

Published: 23/01/2020

Received: 04/07/2019

Copyright: (c) the authors; licensee ecancermedicalscience. This is an Open Access article distributed under the terms of the Creative Commons Attribution License (http:// creativecommons.org/licenses/by/3.0), which permits unrestricted use, distribution, and reproduction in any medium, provided the original work is properly cited. 


\section{Methods}

\section{Patients and $B C$ subtypes}

Retrospective analysis of patients with invasive BC was performed, including all the women treated between January 1997 and August 2018 at Nuestra Señora de la Esperanza Cancer Centre in the Pontificia Universidad Católica de Chile or the Red de Salud UC-Christus Health Network, that were registered in our database. The Scientific and Ethics Committee approved this study. Assessed variables included: age at diagnosis, reason for attending the clinic (RAC) (this is the reason why patients consulted the clinic), dividing those who were motivated by symptoms or signs and those who were motivated by routine mammography findings, TNM (Tumor, Nodes, Metastases) stage [5], histological grade (HG) and tumour subtype. Mammograms referred as RAC were performed as BC screening. Patients who consulted for symptoms or signs of $B C$ also had mammograms as part of workout, but those were not their reason for consultation. Survival rates were calculated and censored according to last follow-up date. Tumour subtypes were defined as Luminal A/B, Human Epidermal Growth Factor Receptor type-2 (HER2) [6]-enriched or Triple Negative (TN) as described [7].

\section{Statistical analysis}

According to their distribution, data were presented as average \pm standard deviation or median (range). Categorical variables are presented as frequency or percentage. Continuous variables were compared by Student's t-test, categorical variables were compared by chi-square or Fisher's exact; $p$-value < 0.05 was considered statistically significant. Analyses were performed in SPSS v21 (IBM).

\section{Results}

Medical records from 2,723 BC patients diagnosed between 1997 and 2018 were analysed (Figure 1A). Clinical characteristics of patients are summarised in Table 1. Age at diagnosis during the first (1997-2007) or the second decade (2008-2018) were similar (55.4 \pm 12.9 versus $55.9 \pm 13.2$, respectively; $p=0.34)$. Tumour stage at diagnosis was obtained for 2,470 cases. As expected, most patients were stage I/II (74.4\% combined). Tumour subtype was obtained for 2,286 patients (84\%). Again as expected, the majority were luminal/hormone dependent BCs (Luminal A/B: 81.3\%; Table 1). The RAC was obtained on 1,754 patients $(64.4 \%) ; 36.9 \%(n=648)$ were diagnosed by mammography, the remaining $63.1 \%(n=1,106)$ by symptoms. Especially, \% of stage I was significantly higher among those diagnosed by mammography compared to symptoms ( $58.55 \%$ versus $17 \%, p<0.0001$, Table 2$)$. The proportion of patients diagnosed by mammography in the first versus the second decade were alike ( $p=0.194)$.

Interestingly, we found a progressive and significant increase in stage IV cases over the assessed period (Figure 1B). A Poisson regression model demonstrates a 3\%/year increase in stage I/II/III patients versus a 11\%/year increase in stage IV ( $p=0.0001)$. Solid line in Figure 1B represents a linear regression estimate of a 7\% cumulative increase in stage IV cases in 20 years (predicted) versus the incidence of stage IV BC cases (observed). HG was obtained on 1,284 cases (47.2\%): 17.9\% were HG1 $(n=230), 40 \% \mathrm{GH} 2(n=514)$, and $42.1 \% \mathrm{HG}(n=540)$.

\section{Overall survival}

Median follow-up was 57.5 months (0-237), with 451 deaths (16.6\%). Five-year overall survival (OS) for the entire group was 88.6\%. OS at 5 years was statistically similar between the first and second decade of the study (1997-2007: 88.8\% versus 2008-2018: 88.3\%, $p=0,621$ ). As expected, survival rates were significantly lower on stage IV cases (Figure $1 \mathrm{C}$ ). Conversely, patients diagnosed by mammography had significantly better survival rates versus symptoms: 5 -year survival was $96 \%$ versus $86.1 \%$, respectively, and 10 -year survival was $90.1 \%$ versus $72.4 \%$, respectively ( $p<0.001$; Figure 1D). Five-year, 10-year and 15-year OS values by stage or tumour subtype are summarised in Supplementary Table S1. In addition, OS rates by BC subtype, Luminal versus non-luminal, by HER2 status, by stage or HG are shown in Supplementary Figures S1 and S2. 
A

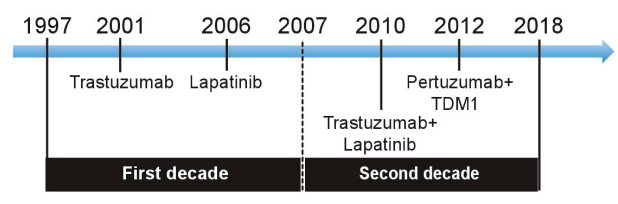

C

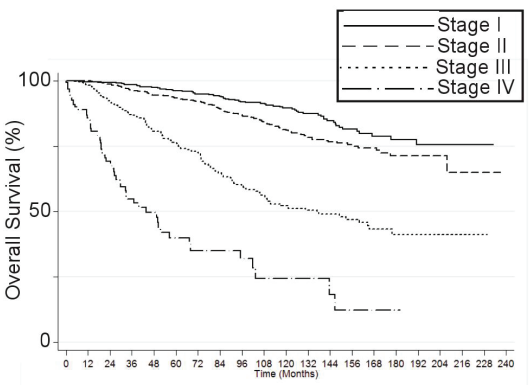

B
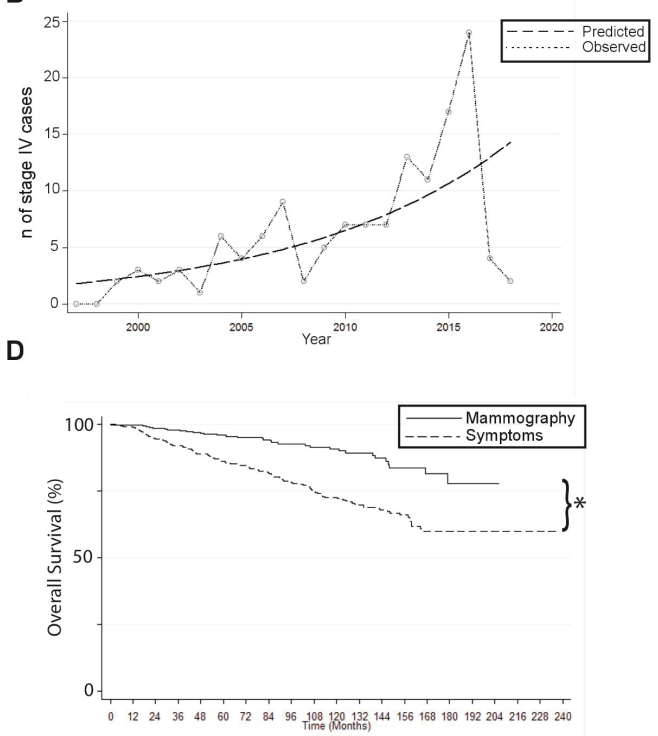

Figure 1. A 20-year experience: main results. (A): Study timeline including year of approval for HER2 BC treatments. (B): Number of stage IV cases over time. Chart compares predicted versus observed cases. (C): Overall survival rates on patients by tumour stage. (D): Overall survival rates on patients diagnosed by screening mammography or by symptoms. ( ${ }^{*} p<0.05$ by log-rank).

Table 1. Clinical characteristics of breast cancer patients diagnosed in the 1997-2018 period.

\begin{tabular}{|l|c|}
\hline Age at diagnosis, average \pm SD & $55.7 \pm 13.13$ years \\
\hline Tumour stage at diagnosis & $\mathrm{N},(\%)$ \\
\hline Stage I & $878(35.5 \%)$ \\
\hline Stage II & $961(38.9 \%)$ \\
\hline Stage III & $496(20.1 \%)$ \\
\hline Stage IV & $135(5.5 \%)$ \\
\hline \multicolumn{1}{|c|}{ BC tumour subtype } & $\mathrm{N},(\%)$ \\
\hline Luminal A & $1,083(47.4 \%)$ \\
\hline Luminal B & $776(33.9 \%)$ \\
\hline HER2-enriched & $160(7.0 \%)$ \\
\hline Triple Negative & $267(11.7 \%)$ \\
\hline
\end{tabular}

Table 2. Tumour stage at diagnosis by reason for attending the clinic.

\begin{tabular}{|l|c|c|}
\hline Tumour stage & Mammography \% & Symptoms \% \\
\hline Stage I & $58.5 \%$ & $17.45 \%$ \\
\hline Stage II & $31.7 \%$ & $45.3 \%$ \\
\hline Stage III & 7.90 & 29.7 \\
\hline Stage IV & 1.77 & $7.52 \%$ \\
\hline
\end{tabular}


Table S1. Five, ten and fifteen year overall survival (OS) by tumor stage or BC subtype.

\begin{tabular}{|l|c|c|c|}
\hline & 5-year OS & 10-year OS & 15-year OS \\
\hline \multicolumn{1}{|c|}{ Tumor stage } & $\%(n)$ & $\%(n)$ & $\%(n)$ \\
\hline Stage I $(n=878)$ & $96.2(476)$ & $89.6(228)$ & $77.6(49)$ \\
\hline Stage II $(n=961)$ & $93.4(480)$ & $80.3(249)$ & $71.4(53)$ \\
\hline Stage III $(n=496)$ & $75.7(198)$ & $51.1(84)$ & $41.1(17)$ \\
\hline Stage IV $(n=135)$ & $40(18)$ & $24.4(4)$ & - \\
\hline \multicolumn{1}{|c|}{ BC subtype } & $\%(n)$ & $\%(n)$ & $\%(n)$ \\
\hline Luminal A ( $n=1,083)$ & $93.8(515)$ & $83.7(25)$ & $70.5(46)$ \\
\hline Luminal B $(n=456)$ & $86.5(206)$ & $68.3(57)$ & $58.7(6)$ \\
\hline HER2-enriched $(n=160)$ & $76.8(57)$ & $63.6(18)$ & $63.6(3)$ \\
\hline Triple Negative $(n=271)$ & $85.2(124)$ & $73.7(49)$ & $58.6(7)$ \\
\hline
\end{tabular}
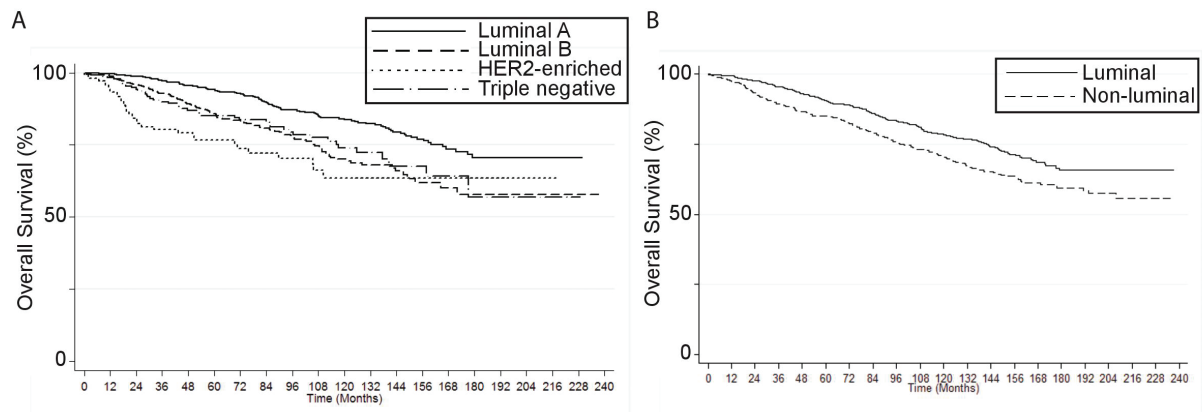

C

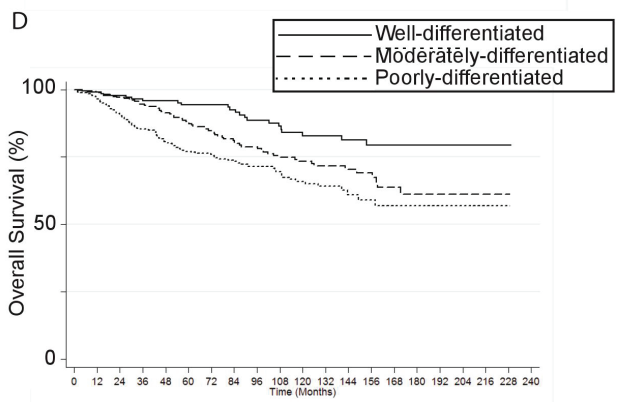

Figure S1. Breast cancer overall survival by (A) molecular subtype, (B) luminal vs non-luminal, (C) HER2 status, and (D) histological grade (Kaplan Meier).

\section{Multivariate analysis}

Supplementary Table S2 shows the results of the multivariate analysis for OS. Period of study was not included in this analysis since it showed no differences between the 2 compared decades. Since HG is included into our definition of BC subtype it was also excluded from analysis. Age, RAC, stage and BC subtype were associated to OS. As expected, stage III/IV mortality rates were significantly higher than stage I. In addition, mortality rates of HER2-enriched were 1.98-fold higher versus luminal-A. Finally, symptomatic patients had a 1.77-fold higher estimated mortality against mammography. Supplementary Table S3 compares clinicopathological variables between patients consulting for altered mammograms and those with signs or symptoms of BC. 

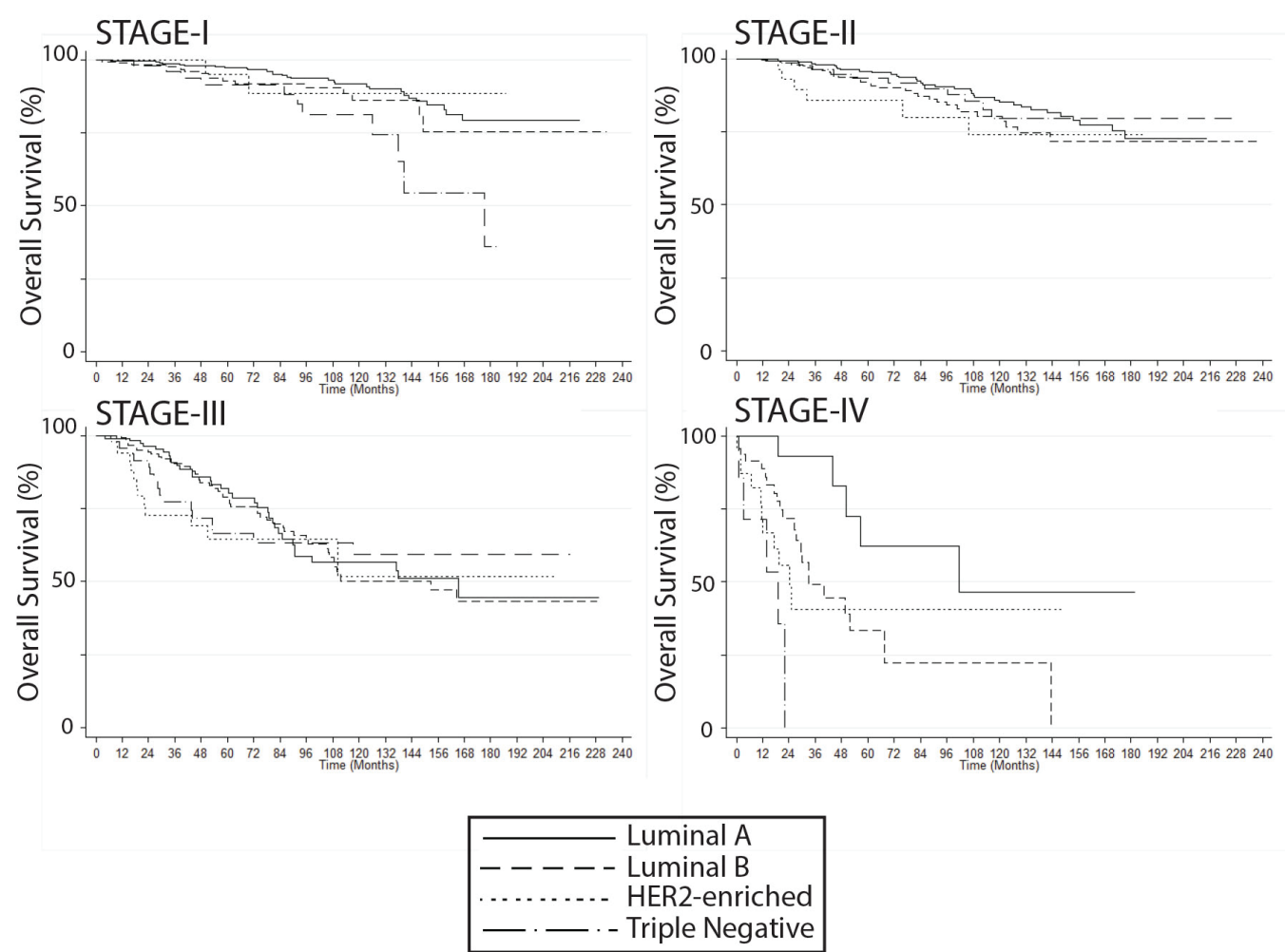

Figure S2. Breast cancer overall survival by molecular subtype, stratified by stage at diagnosis (Kaplan Meier).

Table S2. Multivariate analysis of survival: Cox Regression.

\begin{tabular}{|l|c|c|c|c|}
\hline & Hazard Ratio & $p$-value & \multicolumn{2}{|c|}{ Cl: 95\% } \\
\hline Age & 1.021 & 0.001 & 1.009 & 1.033 \\
\hline Stage I & (ref.) & - & - & - \\
\hline Stage II & 0.958 & 0.868 & 0.580 & 1.584 \\
\hline Stage III & 2.759 & 0.000 & 1.689 & 4.507 \\
\hline Stage IV & 13.017 & 0.000 & 7.139 & 23.734 \\
\hline Luminal A & (ref.) & - & - & - \\
\hline Luminal B & 1.338 & 0.143 & 0.906 & 1.977 \\
\hline HER2 enriched & 1.978 & 0.040 & 1.031 & 3.795 \\
\hline Triple negative & 1.460 & 0.134 & 0.890 & 2.395 \\
\hline RAC* Symptoms & 1.765 & 0.011 & 1.1408 & 2.7305 \\
\hline
\end{tabular}

Abbreviations: $\mathrm{RAC}=$ Reason for Attending the Clinic, $\mathrm{Cl}$ 95\%= Confidence Interval 95\%

Ref.: this value was used as reference 
Table S3. Comparison of clinicopathological variables between patients consulting for altered mammograms and those with signs or symptoms of BC.

\begin{tabular}{|l|c|c|c|}
\hline \multicolumn{1}{|c|}{$\mathrm{N}:$ 1,754 patients } & $\begin{array}{c}\text { Mammograms } \\
(\mathrm{N}: \text { 648) }\end{array}$ & $\begin{array}{c}\text { Signs or symptoms } \\
\text { (N: 1,106) }\end{array}$ & p-value \\
\hline Age at diagnosis, average \pm SD & $56.5 \pm 10.6$ years & $54.5 \pm 14.8$ years & 0.002 \\
\hline Tumor stage at diagnosis & $\mathrm{N},(\%)$ & & \\
\hline Stage I & $363(58.5 \%)$ & $181(17.5 \%)$ & $<0.0001$ \\
\hline Stage II & $197(31.8 \%)$ & $470(45.3 \%)$ & \\
\hline Stage III & $49(7.9 \%)$ & $308(29.7 \%)$ & \\
\hline Stage IV & $11(1.8 \%)$ & $78(7.5 \%)$ & \\
\hline BC tumor subtype & $\mathrm{N},(\%)$ & & \\
\hline Luminal A & $355(62.9 \%)$ & $368(38.6 \%)$ & $<0.0001$ \\
\hline Luminal B & $133(23.6 \%)$ & $350(36.7 \%)$ & \\
\hline HER2-enriched & $27(4.8 \%)$ & $73(7.7 \%)$ & \\
\hline Triple Negative & $49(8.7 \%)$ & $163(17.1 \%)$ & \\
\hline
\end{tabular}

\section{Discussion}

Since their introduction in the early 1980s the widespread use of mammography screenings have demonstrated a concomitant (and expected) increase in early-stage BC incidence. This has been confirmed by demographic data from the Surveillance, Epidemiology and End Results (SEER) database in the United States (US), over the 1973-2008 period [2]. More recently, a study by Verdial et al [8] also analysed the SEER database over the 1973-2013 period and further confirmed these findings, but also showed a decline in overall BC incidence in the 1998-2003 period followed by stabilisation in the 'post-2003'-era. This study also shows that the increase in BC incidence is observed among women aged 50-80 years and establishes that median age for BC diagnosis has risen to 61 years in the US. Accordingly, our study found a median age at diagnosis $n=55$ years that remained unchanged over the 1997-2018 period. However, we demonstrate no changes in early stage BC diagnosis and a surprising increase in stage IV BC cases over the assessed period (Figure 1A and 1B). A couple of reasons could explain these discrepancies: first, our study was performed in a private university hospital where early detection strategies were implemented in the early 1990s, therefore were probably observed prior to the time period covered by our study. In addition, attended patients can afford or have better access to preventive screenings. Second, the increase in stage IV cases could be attributed to better stratification derived from improved more sensitive imaging techniques.

\section{Conclusions}

Previous studies demonstrate that patients that come to the clinic after the appearance of signs/symptoms (versus mammography) almost double their risk of death. Conversely, breast neoplasms diagnosed by mammography display better prognosis, survival rates [9] and reduced mortality [10]. Our study confirms these findings and supports the efficacy of diagnostic screening by mammography in order to reduce BC mortality.

\section{Ethics approval}

The Scientific and Ethics committee at the Pontificia Universidad Catolica de Chile approved this research. Given the retrospective nature of the study no sensitive information was obtained from patients and all data were anonymised. 


\section{Conflict of interest}

The authors declare no conflicts of interest.

\section{Funding statement}

FONDECYT-Iniciacion grant \#11161103 (CS).

\section{References}

1. Siegel RL, Miller KD, and Jemal A (2018) Cancer statistics, 2018 CA Cancer J Clin 68 7-30 https://doi.org/10.3322/caac.21442 PMID: 29313949

2. Bleyer A and Welch HG (2012) Effect of three decades of screening mammography on breast-cancer incidence $N$ Engl J Med 367 1998-2005 https://doi.org/10.1056/NEJMoa1206809 PMID: 23171096

3. Giordano SH, Buzdar AU, and Smith TL, et al (2004) Is breast cancer survival improving? Cancer 100 44-52 https://doi.org/10.1002/ cncr.11859

4. Tan GH, Bhoo-Pathy N, and Taib NA, et al (2015) The Will Rogers phenomenon in the staging of breast cancer - Does it matter? Cancer Epidemiol 39 115-117 https://doi.org/10.1016/j.canep.2014.11.005

5. Edge SB and Compton CC (2010) The American Joint Committee on Cancer: the 7th edition of the AJCC cancer staging manual and the future of TNM Ann Surg Oncol 17 1471-1474 https://doi.org/10.1245/s10434-010-0985-4 PMID: 20180029

6. Acevedo F, Camus M, and Sanchez C (2015) Breast cancer at extreme ages - A comparative analysis in Chile Asian Pacific J Cancer Prev 16 1455-1461 https://doi.org/10.7314/APJCP.2015.16.4.1455

7. Acevedo F, Camus M, and Vial C, et al (2015) [Predictive value of conventional immuno-histochemical biomarkers in breast cancer] Rev Med Chil 143 724-732 https://doi.org/10.4067/S0034-98872015000600005 PMID: 26230555

8. Verdial FC, Etzioni R, and Duggan C, et al (2017) Demographic changes in breast cancer incidence, stage at diagnosis and age associated with population-based mammographic screening J Surg Oncol 115 517-522 https://doi.org/10.1002/jso.24579 PMID: 28194807 PMCID: 5701282

9. Lehtimäki T, Lundin M, and Linder N, et al (2011) Long-term prognosis of breast cancer detected by mammography screening or other methods Breast Cancer Res 13 R134 https://doi.org/10.1186/bcr3080 PMID: 22204661 PMCID: 3326576

10. Mook S, Van 'T Veer LJ, AND Rutgers EJ, et al (2011) Independent prognostic value of screen detection in invasive breast cancer J Natl Cancer Inst 103 585-597 https://doi.org/10.1093/jnci/djr043 PMID: 21350218 